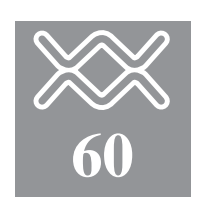

\title{
Posizionare un catetere venoso centrale e prevenire le contestazioni medico-legali
}

\author{
F. Petraruloํ, V. Giancaspro1, F. Fiorini² \\ 'Struttura Complessa di Nefrologia e Dialisi, Ospedale "Di Venere", AUSL BA/04, Bari \\ ${ }^{2} U . O$. Nefrologia e Dialisi, Sanremo, ASL 1 Imperiese
}

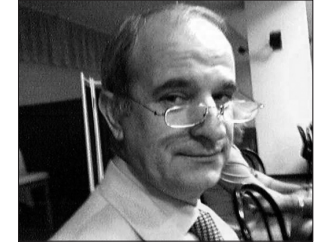

F. Petrarulo
Ua responsabilità professionale medica ha radici molto lontane che ci riportano al 2123 a.C. allorquando nel codice di Hammurabi viene rinvenuta la più antica testimonianza scritta riguardante la responsabilità del medico che con il suo operato determinava la morte del paziente.

In ogni attività sanitaria c'è un momento di rischio professionale: la verifica del rapporto di causalità tra l'azione o l'omissione del medico e il danno verificatosi è condizione indispensabile per il giudizio sulla responsabilità professionale; infatti l'errore può derivare da omissioni o azioni improprie e può dipendere da negligenza, imprudenza o imperizia medica $(1,2)$.

La negligenza consiste in una disattenzione, una trascuratezza o una mancanza di sollecitudine in una determinata azione.

L'imprudenza consiste nella trasgressione di una regola di condotta dalla quale discende l'obbligo di realizzare una determinata azione (o di non realizzarla) con determinate modalità e cioè senza adottare tutte quelle cautele che lo stato dell'arte e l'esperienza in- segna essere necessarie.

L'imperizia consiste in una insufficiente preparazione scientifico-professionale del medico e discende dalla mancata conoscenza della problematica tecnica che il medico è chiamato di volta in volta a risolvere.

La responsabilità penale del medico può essere dolosa, nel caso di trasgressioni volontarie e coscienti o colposa se per negligenza, imprudenza o imperizia cagiona, senza volerlo, la morte o la lesione personale del paziente (3).

Negli ultimi anni si è assistito a un progressivo aumento dei procedimenti penali e delle richieste di risarcimento anche se ci sono stati continui progressi della scienza medica e affinamento delle tecniche operatorie che avrebbero dovuto portare a una riduzione del contenzioso. Questa tendenza è determinata dalla maggiore consapevolezza dei propri diritti da parte dei pazienti e dalla sensibilizzazione operata dalle associazioni di difesa dei diritti del malato. Le richieste di risarcimento solo in minima parte corrispondono a una colpa professionale effettiva che determina un giudizio conclusivo di colpevolezza (4). In un lavoro viene riportato che solo il $25 \%$ delle denunce si concludono con un risarcimento, e di queste solo il $7 \%$ per malpractice, mentre il restante $18 \%$ per patteggiamenti. Inoltre se nel $50 \%$ dei casi esiste un danno al paziente, ma senza colpa del medico (quindi non risarcibile), ben il $25 \%$ delle denunce risulta infondato.

\section{Posizionamento di CVC e consenso informato}

Il primo obbligo del medico nei confronti del paziente per qualsiasi atto medico e nella fattispecie per il posizionamento del CVC è quello di informarlo in modo chiaro ed esauriente sulla natura e sui rischi dell'atto a cui verrà sottoposto. La necessità pregiudiziale di acquisire l'accordo del soggetto interessato prima di procedere a qualsivoglia intervento, diagnostico, terapeutico o sperimentale risponde a un principio etico ormai consolidato e codificato non solo in norme costituzionali e disposizioni legislative, ma anche in trattati e documenti internazionali. I fondamenti giuridici dell'obbligo di informare il paziente sono due: gli artt. 13 e 32 della Costituzione. Qualora il paziente non sia informato sarebbe violato da un lato il suo diritto all'autodeterminazione e, dall'altro, il suo diritto a non essere sottoposto a trattamenti sanitari contro la sua volontà (5). 
La finalità dell'obbligo di informazione è quello di mettere il paziente in condizione di valutare consapevolmente l'opportunità di sottoporsi o meno al trattamento proposto. Tenuto conto di ciò l'informazione deve comprendere:

- la natura e la portata dell'intervento; - i rischi che comporta, anche se ridotti; - le teoriche possibilità di successo;

- la possibilità di conseguire il medesimo risultato attraverso altri interventi e i rischi di questi ultimi.

$\mathrm{Nel}$ caso in cui il medico non informi adeguatamente il paziente sui rischi e sulle possibilità di successo dell'intervento che si appresta a compiere egli è responsabile nei confronti del paziente per l'ipotesi in cui dall'intervento derivino lesioni personali. In questi casi è del tutto irrilevante che l'intervento sia stato eseguito con perizia e senza colpa. Il consenso deve essere espresso personalmente dal soggetto titolare del diritto alla salute salvo due eccezioni date dalla non capacità di intendere e di volere e cioè nel caso del minore e dell'incapace. Al di fuori di queste ipotesi regolamentate a livello giuridico in maniera inequivocabile l'intervento su un soggetto maturo e responsabile potrà realizzarsi solo con il suo consenso salvo il caso in cui questi versi in stato di incoscienza e ricorra uno stato di necessità o urgenza in cui il rinvio dell'intervento non potrebbe essere effettuato se non con grave rischio per il paziente (stato di necessità).

Il carattere personale e fiduciario del rapporto medico-paziente non richiederebbe la manifestazione scritta del consenso, salvo alcuni casi previsti dalla legge (ad esempio la donazione di sangue, la trasfusione di sangue ed emoderivati, in caso di sperimentazione e, ancora, in relazione alla particolare invasività delle prestazioni diagnostiche e/o terapeutiche e/o delle loro conseguenze). In ogni caso il consenso andrebbe richiesto e riformulato per ogni singolo atto terapeutico o diagnostico. Un consenso prestato sulla base di un'informazione carente o scorretta è un consenso viziato e perciò inefficace. La prestazione del consenso non è soggetta ad alcuna forma particolare (nel nostro ordinamento vige il principio della libertà del negozio giuridico). È consigliabile acquisire un consenso scritto dove viene particolareggiata la natura della procedura e dove vengono descritti i rischi e le possibilità di successo della metodica secondo lo stato dell'arte. Il modulo di consenso informato deve essere letto dal paziente e dal medico al paziente e quindi datato e firmato da entrambi solo dopo che il medico si sia sincerato che il paziente abbia percepito la situazione clinica in cui si trova e le possibili alternative terapeutiche in modo tale da poter avere una effettiva possibilità di scelta. In ogni caso il consenso scritto deve essere preceduto da adeguata informazione per garantire la piena consapevolezza del paziente e la sua reale partecipazione al processo terapeutico.

\section{Posizionamento del CVC}

Le tecniche standard di posizionamento del CVC prevedono un isolamento chirurgico del vaso da incannulare o una venipuntura percutanea. Il metodo chirurgico elimina i rischi legati alla incannulazione a cielo coperto ma per la maggiore invasività dell'intervento e per l'inconveniente della notevole difficoltà se non della impossibilità di un eventuale successivo incannulamento della vena è indicato solo in casi clinici particolarmente "difficili" e in emodialisi è raramente utilizzato.

La venipuntura percutanea con l'inserimento dei CVC secondo la tecnica di Seldinger è il metodo più utilizzato. Poiché la puntura alla cieca non è priva di rischi è stato suggerito l'utilizzo dell'ecografia per localizzare il decorso del vaso. L'incannulamento ecoguidato riduce la possibilità di errore; infatti è importante conoscere la "situazione anatomica" di ogni paziente cui deve essere posizionato il catetere venoso (6, 7). Per il trattamento emodialitico la vena giugulare interna (VGI) destra è generalmente riconosciuta come il vaso di elezione per il posizionamento del CVC. Nel caso della puntura della VGI, a fronte di una normale misura e posizione della VGI destra riscontrato nel $61 \%$ dei casi, nel restante $39 \%$ ci possono essere alterazioni (8). I dati della letteratura sono univoci e sottolineano l'importanza di una valutazione non invasiva del vaso mediante esame ecografico. L'ecografia con sonde lineari da 7.5-9 Mhz consente di visualizzare le strutture vascolari superficiali e profonde, di determinare la corretta posizione dei vasi, di individuare il vaso da incannulare, di effettuare la puntura venosa sotto controllo e di visualizzare la penetrazione dell'ago guida nel vaso.

La differenza tra incannulazione ecoguidata, e non, è notevole. La percentuale di successo e di riuscita al $1^{\circ}$ tentativo è superiore per l'incannulamento ecoguidato (99\% vs 90\%), mentre la percentuale di complicanze è inferiore nell'incannulamento ecoguidato: percentuale di puntura arteria (1.7 vs $8 \%$ ), ematoma $(0.2$ vs $3 \%)(9,10)$. Pertanto la puntura ecoguidata in mani esperte permette di eseguire la manovra con la massima sicurezza, di ridurre al minimo la percentuale di insuccesso o di complicanza e quindi di evitare possibili contestazioni medico-legali.

\section{Preparazione del piano di lavoro sterile e manovre in sterilità}

Una volta ottenuto il consenso informato, a paziente supino si procede a disinfettare la superficie del collo e a preparare il campo operatorio in sterilità. L'inserzione dell'ago guida viene preceduta da anestesia locale (xilocaina $1 \%$ ) nel punto di inserzione dell'ago precedentemente identificato con una scansione ecografica. Gel sterile viene applicato sul trasduttore che a sua volta viene coperto con una guaina sterile in materiale plastico. Al trasduttore viene applicato un supporto sterile di plastica al quale viene agganciato l'ago guida. La cute viene bagnata con soluzione sterile salina e il trasduttore viene posto in corrispondenza del triangolo del Sedillot. Usando la tecnica ecografica in "real time" la vena può essere facilmente incannulata mediante la visualizzazione diretta dell'entrata dell'ago guida nella vena $(9,11)$.

Durante tutta la durata della procedura di incannulamento del vaso e di posizionamento del CVC è pressoché indispensabile il monitoraggio continuo 


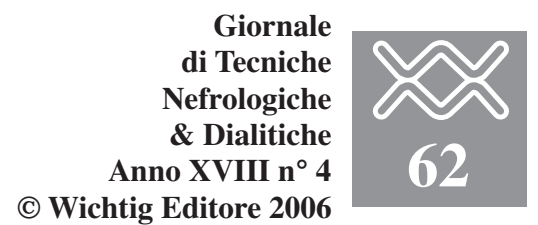

elettrocardiografico a causa della segnalazione di insorgenza di aritmie stimolate dal posizionamento del filo guida metallico (12): se disponibile è ottimale l'utilizzo di un defibrillatore dotato di monitor per la visualizzazione del tracciato ECG in "real time" che può rivelarsi risolutivo in caso di necessità di cardioversione.

Dopo aver posizionato il CVC è necessario verificare e documentare il corretto posizionamento dello stesso mediante esame radiografico standard del torace o fluoroscopia o elettrocardiografia endocavitaria (EC-ECG). Il corretto posizionamento dei CVC è importante sia per ottenere dei flussi ematici adeguati necessari a una efficiente depurazione sia per ridurre al minimo le complicanze dovute all'insulto meccanico da parte della punta. L'alloggiamento della punta varia in base al tipo di catetere:

1) I cateteri temporanei, in genere più rigidi e dalla estremità appuntita, vanno posizionati con punta in cava superiore, $2-3 \mathrm{~cm}$ al di sopra della giunzione atriocavale; per i cateteri inseriti in vena femorale la punta va posizionata nella vena iliaca comune o nella parte inferiore della vena cava inferiore.

2) I cateteri permanenti in silicone $o$ in poliuretano modificato e con la parte terminale smussa e non rigida vanno posizionati con la punta in corrispondenza della giunzione atrio-cavale o nella parte alta dell'atrio dx; la punta non deve tuttavia entrare in contatto con la parete atriale, né con la valvola tricuspide e il seno coronario. Un CVC deve essere posizionato scegliendo la lunghezza in relazione alla sede di incannulazione scelta e alla corporatura del paziente e basandosi sui reperi anatomici costituiti in particolare dal IV spazio intercostale destro.

\section{La registrazione elettrocardiografia endocavitaria (ECG-EC) continua}

Il controllo ECG-EC si basa sulla registrazione dell'attività elettrica intraatriale e quindi sulle modificazioni dell'onda "P" che presenta una ampiezza maggiore rispetto alla $\mathrm{P}$ dell'ECG di superficie, a seguito dell'impedenza ridotta legata ai tessuti e alla minore distanza dal nodo del seno, sede dell'impulso elettrico. Tale registrazione è ottenuta utilizzando il CVC stesso come un elettrodo esploratore endovascolare, collegandolo alla derivazione V2 o D3 e registrando quindi il tracciato ECG (13). Con il progressivo avvicinamento del tip del catetere all'atrio destro, la deflessione negativa dell'onda $\mathrm{P}$ aumenta progressivamente di ampiezza per diventare difasica quando la punta penetra nell'atrio e invertita, pertanto positiva, nel ventricolo destro o nella vena cava inferiore. Questo controllo rende superfluo quello fluoroscopico durante l'intervento di cateterismo venoso evitando l'esposizione a radiazioni ed ha il vantaggio di essere una metodica semplice, precisa, affidabile, rapida e a costo quasi zero.

\section{Controllo del posizionamento del CVC}

Le linee guida americane (NKF-DOQI) considerano il controllo radiologico indispensabile dopo l'inserzione in vena succlavia e giugulare interna e prima di iniziare la seduta dialitica al fine di confermare la corretta posizione del catetere e di escludere eventuali complicanze (14).

La radiografia del torace antero-posteriore è considerato il "golden standard" nel controllo del posizionamento dei CVC per la facilità di esecuzione e per l'affidabilità nel documentare la posizione finale del catetere e la presenza di un eventuale pneumotorace o altre complicanze pleuroparenchimali. Il controllo radiologico dovrebbe essere eseguito dopo 4 ore e ripetuto dopo 24 ore. Il primo controllo consente di valutare l'esatto posizionamento del catetere e di escludere complicanze precoci, il secondo permette di escludere un pneumotorace tardivo insorgente dopo 12-24 ore (15). Peraltro due autorevoli società scientifiche (la Società Americana e la Società Italiana di Nutrizione Parenterale ed Enterale) hanno pubblicato delle linee guida secondo le quali il controllo radiologico durante il cateterismo sarebbe necessario solo in caso di pun- tura alla cieca non ecoguidata della vena succlavia e della VGI.

Anche la fluoroscopia è ritenuta dalle stesse linee guida necessaria per l'inserzione di CVC tunnellizzati. Peraltro nella pratica routinaria il monitoraggio "on line" fluoroscopico che permetterebbe il controllo durante il cateterismo della posizione della guida metallica, del dilatatore e del catetere è raramente praticato anche a causa della alta esposizione alle radiazioni sia del paziente sia dello staff e per i problemi dei costi derivanti nell'allestire l'apparecchiatura fuoroscopica necessaria. L'indicazione all'impiego della fluoroscopia rimane nei casi di presenza di pace-maker o di filtro cavale, di precedenti CVC o di pregressi tentativi infruttuosi di CVC. In questi casi è inoltre consigliabile eseguire prima dell'intervento di posizionamento del CVC uno studio angiografico per la possibilità di stenosi o occlusioni delle vene centrali. Nei casi clinici non complicati recenti lavori hanno messo in discussione anche l'utilità del controllo radiologico post-inserzione soprattutto nel cateterismo della VGI qualora la manovra venga eseguita con la puntura ecoguidata del vaso e con registrazione ECG-EC; nel 99\% dei casi si ottiene un corretto posizionamento del CVC con rischio di pneumotorace ridotto a zero. In ogni caso il controllo radiografico consente di verificare il corretto decorso del CVC escludendo eventuali torsioni o inginocchiamenti e in caso di incannulamento a sinistra l'aspetto caratteristico definito a "tenda" indicativo di una deformazione della parete della vena cava superiore determinata dalla punta del catetere (16).

\section{CONCLUSIONI}

In breve, la prevenzione delle possibili contestazioni medico-legali conseguenti al posizionamento di un CVC passa attraverso una iniziale ed esaustiva informazione al paziente circa le procedure che andremo a intraprendere e con quanto ne può conseguire. Un indispensabile obbligo di mezzi da parte del medico esecutore della metodica (idonea preparazione teorica e pratica, 
idonea strumentazione ecc.) deve permettere di gestire tutte le procedure necessarie ad eseguire tale manovra in modo corretto minimizzando, se non annullando, la possibilità di incorrere in complicanze, in "errori" perseguibili penalmente.

\section{BIBLIOGRAFIA}

1. Puccini. Istruzioni di Medicina Legale. Casa Ed. Ambrosiana, Milano 1998.

2. Bilancetti M. La responsabilità penale e civile del medico. CEDAM, Padova 1998.

3. Prevosto A. La responsabilità penale nell'esercizio dell'attività medicochirurgica e il grado di colpa. L'Indice Penale, 1982.

4. Sentenza emessa dal Giudice Monocratico del Tribunale di Nola, dr Polizzi, in data 30.06.2004 e depositata in data 30.09.2004.

5.

Fiorini F, Palombo G, Ciliberti R. Consenso informato al posizionamento dei cateteri venosi centrali: una proposta operativa. Giorn It Nefr 2004; 5 : 463-8.

6.

Denys BG, Uretsky BF, Reddy PS. Ultrasound-assisted cannulation of the internal jugular vein. A prospective comparison to the external landmarkguided technique. Circulation 1993; 87 (5): 1557.

7. Dionisio P, Cavatorta F, Zollo A, et al. The placement of central venous catheters in hemodialysis: role of endocavitary electrocardiographic trace. Case report and literature review. J Vasc Access 2001; 2: 80-8.

8. Bing-Shi Lin, Chi-Woon, et al. Anatomical variation of the internal jugular vein and its impact on temporary haemodialysis vascular access: an ultrasonographic survey in uraemic patients. Nephrol Dial Transplant 1998; 13: $134-8$.

9. Conz PA, La Greca G. Cannulation of the internal jugular vein: comparison of the classic Seldinger technique and an ultrasound guide method. J Nephrol 1997; 87: 1557.

10. Kairaitis L, et al. Outcome and complications of temporary hemodialysis catheters. Nephrol Dial Transp lant 1999; 14: 1710-4.
11. Czepizak CA, O'Callaghan JM, Venous B. Evaluation of formula por optimal positioning of central venous catheters. Chest 1995; 107: 1662-4.

12. Eisen LA, Narasimhan M, Berger JS, et al. Mechanical complications of central venous catheters. J Intensive Care Med 2006; 21: 40.

13. Cavatorta F, Fiorini F, Campisi S, et al. Ultrasound-guided cannulation and endocavitary electrocardiography in placement of central venous catheters. Clin Nephrol 1999; 52: 91-193.

14. Schwab S, Esarab A, Beathard NKF-DQI clinical practis guidelines for vascular access. National Kidney Foundation-Dialysis Outcomes Quality Initiative. Am kidney Dis 1997; 30 (Suppl): S50-91.

15. Cavatorta F, Zollo A, Dionisio P, Galli F. Il posizionamento dei cateteri venosi centrali: modalità di controllo. In: L'accesso vascolare in emodialisi (3). Ed. Accademia Nazionale in Medicina 2005.

16. CVC-partner. Controlling the placement of central venous catheters. N. 2 Ed. Braun Hospital Care 2002. 